\title{
Magnetic microstructure of bacterial magnetite by electron holography
}

\author{
MARTHA R. McCARTNEY ${ }^{1}$, ULYSSES LINS ${ }^{2}$, MARCOS FARINA ${ }^{3}$, PETER R. BUSECK ${ }^{4}$ and \\ RICHARD B. FRANKEL ${ }^{5}$
}

\begin{abstract}
${ }^{1}$ Center for Solid State Science, Arizona State University, P.O. Box 871704, Tempe, AZ 85287-1704,
Arizona, USA. e-mail: molly.mccartney@asu.edu

${ }^{2}$ Instituto de Microbiologia Professor Paulo de Góes, Universidade Federal do Rio de Janeiro, Rio de Janeiro, Brazil

${ }^{3}$ Instituto de Ciências Biomédicas, Universidade Federal do Rio de Janeiro, Rio de Janeiro, Brazil

${ }^{4}$ Departments of Geological Sciences and Chemistry/Biochemistry, Arizona State University, Tempe, Arizona, USA

${ }^{5}$ Department of Physics, California Polytechnic State University, San Luis Obispo, California, USA. e-mail: rfrankel@calpoly.edu
\end{abstract}

\begin{abstract}
A brackish lagoon at Itaipu, Brazil, contains magnetotactic bacteria with unusually large magnetite magnetosomes (lengths 100-200 nm). The micromagnetic structures of the magnetosomes from two different coccoid organisms from the lagoon have been determined by electron holography. The results are consistent with single-magnetic-domain structure in the elongated magnetosomes from one organism and metastable, singlemagnetic-domain structure in the larger, more equi-axed, magnetosomes from the other organism. The results are consistent with theoretical predictions of the transition dimension between stable and metastable single-domain structure in magnetite.
\end{abstract}

Key-words: magnetite, magnetotactic bacteria, electron holography, magnetic microstructure, single-magnetic domain.

\section{Introduction}

Magnetotactic bacteria orient and migrate along geomagnetic field lines. Each cell contains a permanent magnetic dipole comprising membrane-enclosed magnetite $\left(\mathrm{Fe}_{3} \mathrm{O}_{4}\right)$ or greigite $\left(\mathrm{Fe}_{3} \mathrm{~S}_{4}\right)$ nanocrystals known as magnetosomes (Gorby et al., 1988). The magnetosomes are typically organized in chain structures and have specific crystal morphologies within each cell type (Bazylinski \& Frankel, 2000). In some bacterial strains, magnetite crystals in magnetosomes are cuboctahedra comprising [100] and [111] forms. In other bacterial strains, the magnetite crystals are elongated along a [111] axis parallel to the chain direction and have idealized habits comprising [100], [111], and [110] forms. In these habits, the six, eight, and twelve symmetry-related faces of the respective forms expected for the face-centered $(\mathrm{Fd} 3 \mathrm{~m})$ spinel structure are not equally developed (Devouard et al., 1998). The elongated magnetite crystals are typically 40 to $100 \mathrm{~nm}$ long, within the stable, single-magnetic-domain (SD) size range.

At least four magnetite-producing, coccoid, bacterial morphotypes, Itaipu 1, 2, 3 and 4 (Spring et al., 1998) and a rarely observed rod-shaped bacterium (Lins \& Farina, 1998), occur in a brackish lagoon at Itaipu, located on the coast of Brazil, north of Rio de Janeiro. Morphotype Itaipu 1, the largest organism, is a diplococcus with two chains of magnetosomes; the magnetosome crystals have roughly square projections and lengths up to 200 nm (Farina et al., 1994; Spring et al., 1998). These are the largest magnetosome crystals yet reported. 


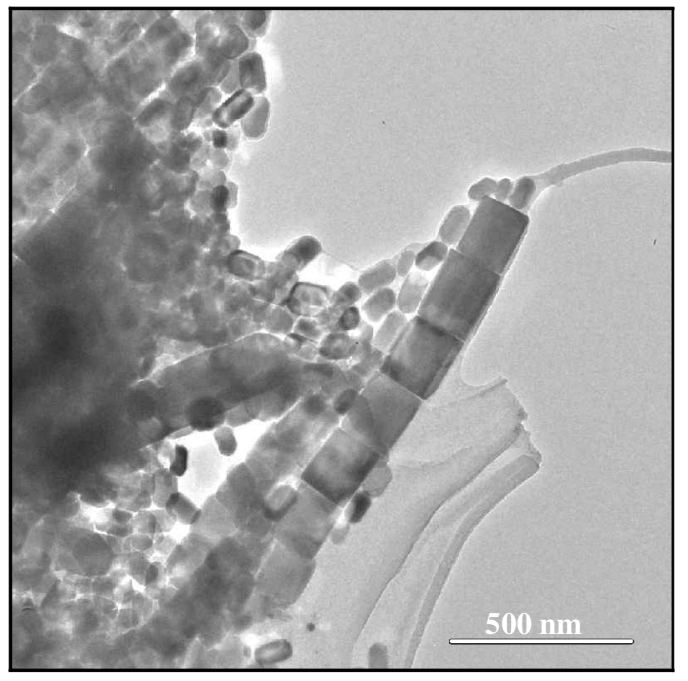

Fig. 1. TEM image of larger, equi-axed magnetosomes from Itaipu 1 and smaller, elongated magnetosomes from Itaipu 3 coccoid, magnetotactic bacteria.

Itaipu 2 and 4 are smaller cells containing magnetosome crystals that are smaller than but with similar projections to those in Itaipu 1. Itaipu 3 is a coccus with magnetosome crystals that are elongated (length/width $\sim 2$ ) along the chain direction, with lengths up to $100 \mathrm{~nm}$ and prominent corner facets. As the SD to non-SD transition dimension in magnetite is not well understood and varies with axial ratio (Dunlop \& Özdemir, 1997), it is uncertain whether the magnetite crystals from Itaipu 1 and Itaipu 3 are stable SD or metastable single domains (MSD) with SD structure resulting from magnetostatic interactions between crystals in the magnetosome chains. Here we report on the micromagnetic structure of the large magnetite crystals in morphotype Itaipu 1 and the elongated magnetite crystals in Itaipu 3 determined by off-axis electron holography $(\mathrm{EH})$.

\section{Methods}

Itaipu 1 and Itaipu 3 cells were collected and whole cells, or magnetosomes extracted from disrupted cells, were deposited on TEM grids. In the disruption process, Itaipu 1 and Itaipu 3 magnetosomes were mixed together. EH, HRTEM, and ED measurements were carried out as previously reported (Dunin-Borkowski et al., 1998a; Devouard et al., 1998). Magnetosomes from Itaipu 1 and 3, extract- ed from disrupted cells, are shown in Fig. 1. While isolated single chains of the elongated Itaipu 3 magnetosomes were found on the TEM grid, chains of Itaipu 1 magnetosomes were always found with some Itaipu 3 magnetosomes attached. Single magnetosomes of either type were not found.

In off-axis electron holography, the sample is positioned so that it covers approximately half the field of view, and a charged electrostatic biprism causes the electron wave that has passed through the specimen to overlap with a wave that has passed only through vacuum, which serves as a reference wave. The resulting hologram is an interference pattern in which amplitude information is contained in the relative amplitude of the cosine-like fringes and information about the phase shift of the electron wave is contained in their positions (Dunin-Borkowski et al., 2001, this issue).

By recording digital holograms with a slowscan CCD camera, accurate quantification of the phase and amplitude becomes possible (de Ruijter $\&$ Weiss, 1993), unlike a conventional electron microscope image which represents only the intensity of the electron wave and does not contain any phase information.

For a sample with electrostatic potential, V, and magnetic vector field, $\mathbf{B}$, the phase shift, $\Delta \phi$, of the electron wave along a direction $\mathrm{x}$ in the sample perpendicular to the incident beam direction $\mathrm{z}$ can be expressed (Reimer, 1989) as

$$
\Delta \phi(\mathrm{x}, \mathrm{y})=\frac{-2 \pi}{\lambda \mathrm{E}} \frac{\mathrm{E}+\mathrm{E}_{0}}{\mathrm{E}+2 \mathrm{E}_{0}} \quad \mathrm{~V}(\mathbf{r}) \mathrm{dz}+\frac{\mathrm{e}}{\mathrm{h}} \quad \mathbf{B}(\mathbf{r}) \cdot \mathrm{d} \mathbf{S}(1)
$$

where $\lambda, E, E_{0}, e, h$, and $V$ are the electron wavelength, kinetic energy, rest mass energy, charge, Planck's constant, and electrostatic mean inner potential, respectively, and $\mathrm{dS}=\mathrm{dxdz}$. In the absence of induced electric fields, the first integral is equal to the mean inner potential (Gajdardziska-Josifovska et al., 1993) times the sample thickness. The second integral picks out the components of the magnetic field perpendicular to the incident-beam direction, i.e., those that normally correspond to the components in the plane of the sample.

In order to separately analyze the $3 \mathrm{D}$ shape and the magnetic flux of small magnetic crystals, the two contributions from equation (1) are separated by acquiring two holograms between which the magnetization in the sample has been reversed in situ by the application of the magnetic field of the objective lens (Dunin-Borkowski et al., 1998b). The sum of the phases of these two holograms then represents twice the mean inner potential contribution to 

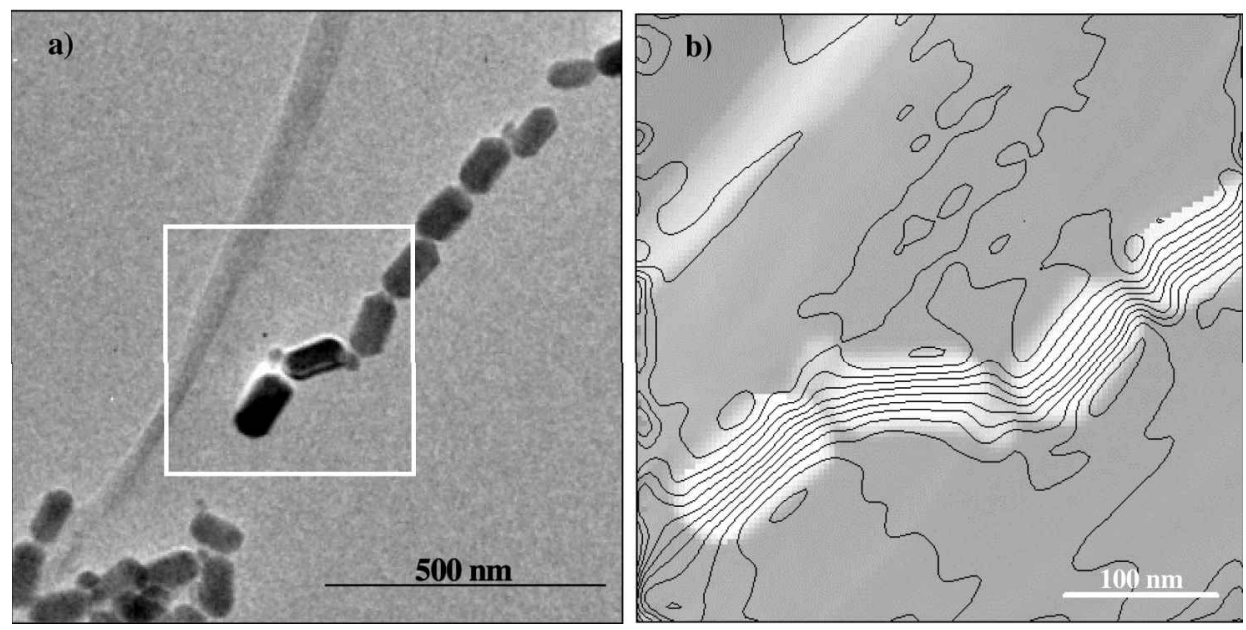

Fig. 2. a) TEM image of a chain of Itaipu 3 magnetosomes. Smaller magnetite crystallites $(<30 \mathrm{~nm})$ are present at kinks in the chain. b) Magnetic flux lines derived from the magnetic contribution to the electron holographic phase image. Contour spacing is $3 \times 10^{-16} \mathrm{~T} \mathrm{~m}^{-2}$. Contours are superimposed on the phase image of projected thickness.

the phase if the magnetization has exactly reversed, while the difference of the phases gives twice the magnetic contribution. The magnetic flux in Itaipu 1 and 3 magnetosomes is reported here; the 3D shape analysis will be reported elsewhere.

\section{Results}

A TEM image of a chain of Itaipu 3 magnetosomes is shown in Fig. 2a. The left end of the chain is kinked due to the presence of small $(<30 \mathrm{~nm})$ crystallites. Fig. 2b shows contours indicating the projected magnetic-flux lines obtained from the magnetic contribution to the electron phase for the end of the chain, superposed on an image of the projected 3D shape of the magnetosomes.

As found previously for magnetosome chains in other bacterial strains (Dunin-Borkowski et al., 1998b), the magnetic flux lines are generally parallel to the chain and show that the Itaipu 3 magnetosome crystals in the chain configuration are SD. However, at the positions of the kinks in the chain the flux lines bend to follow the axis of elongation of the magnetite crystals. The containment of the flux lines within the magnetosomes suggests that the magnetic anisotropy of each crystal is more important than the magnetic anisotropy of the chain as a whole in determining the direction of magnetic flux. Since the magnetocrystalline anisotropy of magnetite is relatively low, the magnetic anisotropy of each magnetosome is dominated by shape an- isotropy. The passage of magnetic flux lines through the $<30 \mathrm{~nm}$ crystallites shows they are also magnetized, although isolated $<30 \mathrm{~nm}$ magnetite crystals would be expected to be superparamagnetic (SPM).

The magnetic flux lines for the chain of Itaipu 1 magnetosomes to the right of center in Fig. 1 are shown in Fig. 3. In this case, some Itaipu 3 crystals have attached themselves to the Itaipu 1 chain. Al-

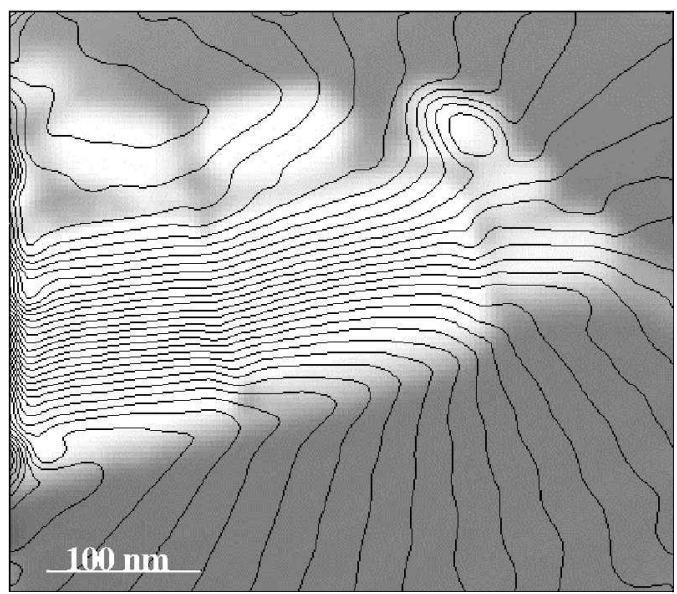

Fig. 3. Magnetic flux lines derived from the magnetic contribution to the electron holographic phase image for the chain of Itaipu 1 magnetosomes shown in Fig. 1. Note flux lines emerging from sides of crystals. Smaller Itaipu 3 crystals appear to cling to the larger magnetosomes. Contour spacing as in Fig. 2. 
though a significant amount of flux can be seen emerging from the sides of the Itaipu 1 magnetosomes, the concentration of flux lines within the crystals that the Itaipu 1 magnetosomes in the chain configuration are SD. The flux density through the Itaipu 3 crystals in Fig. 3 is probably reduced by fringing fields from the adjacent, larger-volume, Itaipu 1 crystals. The circular flux pattern in the small crystal at the left upper end of the chain is probably an artifact due to incomplete reversal or movement of the crystal during the in situ magnetization.

A fortuitous configuration of magnetosomes was also found on the TEM grid that comprised three large Itaipu 1 crystals and three elongated Itaipu 3 crystals, with the Itaipu 1 crystals forming a right angle (Fig. 4). As before, the lines represent the difference in holographic phase images after application of in situ magnetizing fields $(\sim 1 \mathrm{~T})$ along the two directions indicated by the double-headed arrow. This procedure gives the magnetic flux only in case the magnetization has completely reversed between the two holograms, as in Fig. 2 and 3. In the present case, the particular non-linear arrangement indicates the dominance of configurational anisotropy over uniaxial crystalline anisotropy. The direction of the contour lines and the density of the lines indicate the direction of the resultant dif-

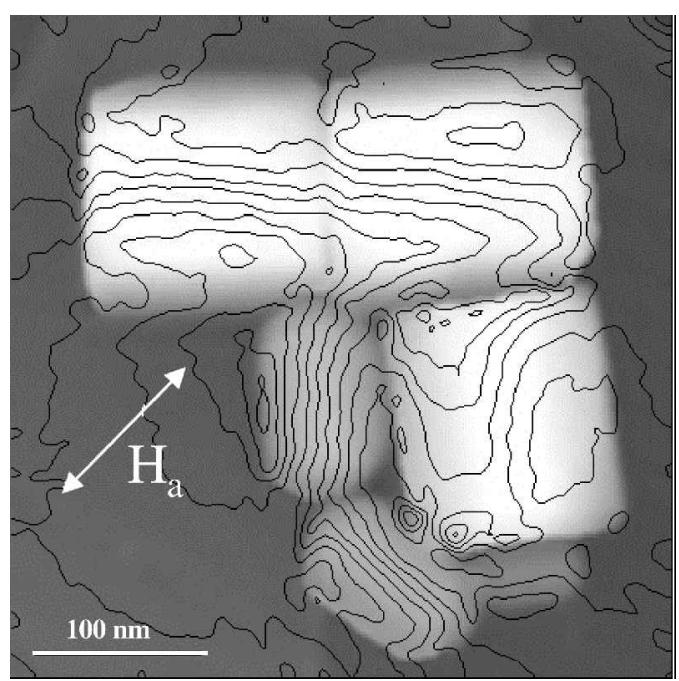

Fig. 4. Contoured image derived from the difference in holographic phase images of Itaipu 1 magnetosomes after application of applied fields as indicated by double-headed arrow. The density of flux lines for smaller Itaipu 3 magnetosomes indicates magnetic reversal for these crystals. See text for discussion of contour pattern in larger Itaipu 1 magnetosomes. ference in magnetization and the cosine of the angle between the magnetizations of the two holograms, respectively.

The containment of the magnetic flux lines parallel to the long axes of the Itaipu 3 magnetosomes, seen in Fig. 4, suggests that these crystals are SD. On the other hand, the difference in magnetization in the upper part of the top two Itaipu 1 crystals is low, indicating that the field directions were similar after application of the in situ magnetizing fields. In the middle of these top two crystals the field contour density is about one half the expected value, indicating that the field directions differ by $60^{\circ}$ rather than $180^{\circ}$. An alternate explanation could be that the crystals contain domain walls parallel to the plane of the sample.

The third Itaipu 1 crystal has the most complicated pattern in the difference image and gives the impression that the field distribution was curved rather than uniform, with areas separated by straight $180^{\circ}$ walls. Also of interest is the apparent lack of fringing fields on the right-hand side of the crystal. This lack indicates significant interior flux closure along that side in both the initial and final magnetization states that would require significant bending of the magnetic flux within the crystal, i.e., it is not SD in this configuration.

\section{Conclusion}

While the magnetic microstructure of the Itaipu 1 crystals in Fig. 4 cannot be unambiguously determined from the difference phase image, it is clear

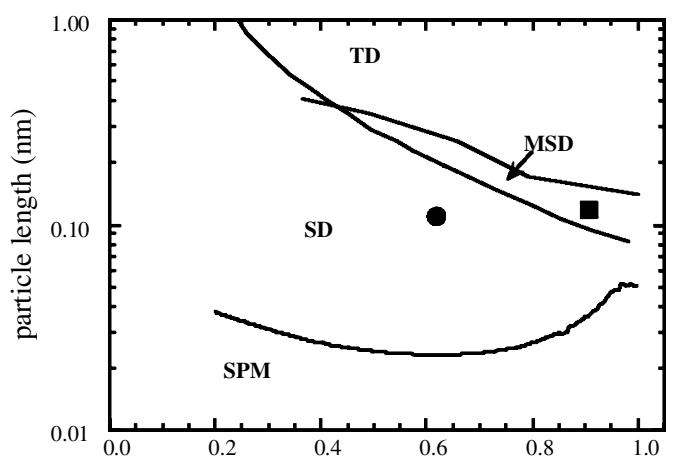

Fig. 5. Theoretical domain-state diagram for magnetite showing the superparamagnetic (SPM), single-domain (SD), two-domain and vortex states (TD), and metastable, single-domain (MSD) stability ranges for parallelepipedshaped particles. Solid square: Itaipu 1 magnetosomes. Solid circle: Itaipu 3 magnetosomes. (After Bazylinski \& Moskowitz, 1997). 
that while Itaipu 3 magnetosomes are SD in both configurations, Itaipu 1 magnetosomes are SD only in the chain configuration where they are magnetized by the neighboring crystals. Fig. 5 shows a theoretical domain-state diagram for magnetite with calculated SPM, SD, TD (two domain and vortex states) and MSD size ranges. (Bazylinski \& Moskowitz, 1997), with solid square and circle indicating Itaipu 1 and Itaipu 3 magnetosomes, respectively. According to Fig. 5, Itaipu 3 magnetosome crystals are predicted to lie in the SD region, in agreement with the results reported here. Itaipu 1 is predicted to lie in the MSD region where the SD size range is extended for materials of low anisotropy by the existence of non-SD "flower-like" states or vortices (Fabian et al., 1996). The curvature of the field lines emerging from the sides of the crystals near the end of the Itaipu 1 magnetosome chain in Fig. 3 is typical of the "flower-like" state. More strikingly, the magnetic configurations of the nonlinear arrangement of Itaipu 1 magnetosomes in Fig. 4, which are certainly more complicated than a simple vortex, exhibits neither stable SD nor TD states, in agreement with the theoretical prediction. This work illustrates the opportunities afforded by $\mathrm{EH}$ analysis of nanoscale biogenic minerals to determine their magnetic microstructures with sizes close to the domain-state transition dimension for comparison with theoretical calculations.

Acknowledgments: We thank Bruce Moskowitz for discussions. The electron holography was performed at the Center for High-Resolution Electron Microscopy at ASU. RBF and PRB acknowledge support from the National Science Foundation. M. Farina and U. Lins acknowledge support from the CNPq (PRONEX) Brazilian Agency.

\section{References}

Bazylinski, D.A. \& Frankel, R.B. (2000): Magnetic ironoxide and iron-sulfide minerals within microorganisms. In Baeuerlein, E., (ed): Biomineralization: From Biology to Biotechnology and Medical Application, pp. 25-46. Weinheim: Wiley-VCH.

Bazylinski, D.A. \& Moskowitz, B.M. (1997): Microbial biomineralization of magnetic iron minerals. In Banfield, J.F. \& Nealson, K.H. (eds.): Geomicrobiology: Interactions between microbes and minerals. Washington, D. C.: Mineralogical Society of America, 35, 181-223. de Ruijter, W.J. \& Weiss, J.K. (1993): Detection limits for quantitative off-axis electron holography. Ultramicroscopy, 50, 269-283.

Devouard, B., Pósfai, M., Hua, X., Bazylinski, D.A., Frankel, R.B., Buseck, P.R. (1998): Magnetite from magnetotactic bacteria: Size distributions and twinning. Amer. Mineral., 83, 1387-1399.

Dunin-Borkowski, R.E., McCartney, M.R., Frankel, R.B., Bazylinski, D.A., Pósfai, M., Buseck, P.R. (1998a): Magnetic microstructure of magnetotactic bacteria by electron holography. Science, 282, 1868-1870.

Dunin-Borkowski, R.E., McCartney, M.R., Pósfai, M., Frankel, R.B., Bazylinski, D.A., Buseck, P.R. (2001): Off-axis electron holography of magnetotactic bacteria: magnetic microstructure of strains MV-1 and MS-1. Eur. J. Mineral., 13, 671-684.

Dunin-Borkowski, R.E., McCartney, M.R., Smith, D.J., Parkin, S.S.P. (1998b): Towards quantitative electron holography of magnetic thin films using in situ magnetization reversal. Ultramicroscopy, 74, 61-73.

Dunlop, D. J. \& Özdemir, Ö. (1997): Rock Magnetism: Fundamentals and Frontiers. Cambridge, UK: Cambridge Univ. Press.

Fabian, K., Kirschner, A., Williams, W., Heider, F., Leibl, T., Huber, A. (1996): Three-dimensional micromagnetic calculations for magnetite using FFT. Geophys. J. Int'l., 124, 89-104.

Farina, M., Kachar, B., Lins, U., Broderick, R., Lins de Barros, H.G.P. (1994): The observation of large magnetite $\left(\mathrm{Fe}_{3} \mathrm{O}_{4}\right)$ crystals from magnetotactic bacteria by electron and atomic force microscopy.J. Microsc., 173, 1-8.

Gajdardziska-Josifovska, M., McCartney, M.R., de Ruijter, W.J., Smith, D.J., Weiss, J.K., Zuo, J.M. (1993): Accurate measurements of mean inner potential of crystal wedges using digital electron holograms. Ultramicroscopy, 50, 285-299.

Gorby, Y.A., Beveridge, T.J., Blakemore, R.P. (1988): Characterization of the bacterial magnetosome membrane. J. Bacteriol., 170, 834-841.

Lins, U. \& Farina, M. (1998): Magnetosome size distribution in uncultured rod-shaped bacteria as determined by electron microscopy and electron spectroscopic imaging Microsc. Res. Tech., 42, 459-464.

Reimer, L. (1989): Transmission Electron Microscopy. Berlin: Springer, p. 37.

Spring, S., Lins, U., Amann, R., Schleifer, K.-H., Ferreira, L.C.S., Esquivel, D.M.S., Farina, M. (1998): Phylogenetic affiliation and ultrastructure of uncultured magnetic bacteria with unusually large magnetosomes. Arch. Microbiol., 169, 136-147. 